\title{
Electronic cigarette use and smoking initiation among youth: a longitudinal cohort study
}

\author{
David Hammond PhD, Jessica L. Reid MSc, Adam G. Cole MSc, Scott T. Leatherdale PhD
}

Cite as: CMAJ 2017 October 30;189:E1328-36. doi: 10.1503/cmaj.161002

\begin{abstract}
BACKGROUND: The influence of ecigarette use on smoking initiation is a highly controversial issue, with limited longitudinal data available for examining temporal associations. We examined e-cigarette use and its association with cigarette-smoking initiation at 1-year follow-up within a large cohort of Canadian secondary school students.
\end{abstract}

METHODS: We analyzed data from students in grades 9-12 who participated in 2 waves of COMPASS, a cohort study of purposefully sampled secondary schools in Ontario and Alberta, Canada, at baseline (2013/14) and 1-year followup $(2014 / 15)$. We assessed cigarette smoking and e-cigarette use at baseline and follow-up using self-completed surveys. We used generalized linear mixedeffects models to examine correlates of past 30-day e-cigarette use at baseline and smoking initiation between waves within the longitudinal sample.

RESULTS: Past 30-day e-cigarette use increased from 2013/14 to 2014/15 (7.2\% v. $9.7 \%, p<0.001$ ), whereas past 30 -day cigarette smoking decreased slightly (11.4\% v. $10.8 \%, p=0.02$ ). Among the 44163 students evaluated at baseline, past 30-day e-cigarette use was strongly associated with smoking status and smoking susceptibility. In the longitudinal sample ( $n=19130)$, past 30-day use of e-cigarettes at baseline was associated with initiation of smoking a whole cigarette (adjusted odds ratio [OR] 2.12, 95\% confidence interval $[\mathrm{Cl}] 1.68-2.66)$ and with initiation of daily smoking (adjusted OR 1.79, 95\% Cl 1.41-2.28) at follow-up.

INTERPRETATION: E-cigarette use was strongly associated with cigarette smoking behaviour, including smoking initiation at follow-up. The causal nature of this association remains unclear, because common factors underlying the use of both e-cigarettes and conventional cigarettes may also account for the temporal order of initiation.

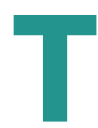

he prevalence of cigarette smoking has declined in Canada, from about 50\% in the 1960 s to $13 \%$ in $2015 .{ }^{1}$ As in most other countries, the majority of Canadian smokers begin smoking during adolescence, and reductions in the prevalence of cigarette smoking in Canada have been achieved primarily through declining rates of smoking initiation among youth. ${ }^{1}$

The emergence of vapourized nicotine products - widely referred to as electronic cigarettes or e-cigarettes - has provided consumers with an alternative means of nicotine intake. The use of e-cigarettes is largely concentrated among adult smokers, most of whom report using e-cigarettes to quit smoking or for the purpose of smoking reduction. ${ }^{2,3}$ However, substantial proportions of youth also report using e-cigarettes. In Canada, about $20 \%$ of youth aged 15-19 years report "ever trying" e-cigarettes, including $14 \%$ of youth nonsmokers, similar to estimates from the United Kingdom and the United States..$^{3-7}$ Although many Canadian youth try e-cigarettes, fewer report regular use. ${ }^{3}$ For example, in a recent study of Canadian youth, only $0.2 \%$ of all youth - and $2 \%$ of those who had tried e-cigarettes - reported daily use. ${ }^{8}$

Studies have consistently shown a strong association between e-cigarette use and cigarette smoking., ${ }^{3,9-11}$ In addition, US studies have suggested that the availability of e-cigarettes has expanded the nicotine market: rather than simply substituting e-cigarettes for cigarette smoking, the total number of youth using any type of nicotine product has increased for the first time in decades. ${ }^{12}$

The extent to which e-cigarette use is causally related to smoking initiation remains a source of considerable debate. To date, 4 studies have examined the temporal order between ecigarette use and smoking initiation. Three studies involving US secondary students found that "ever" users of e-cigarettes at baseline were more likely to report smoking cigarettes 12 months later. ${ }^{13-15}$ In addition, a smaller study of secondary students in California found that students who reported "ever" using e-cigarettes at baseline were more likely to report using cigarettes, cigars, pipes and shisha at follow-up, compared with a matched sample of "never" e-cigarette users. ${ }^{16}$

Although longitudinal studies have been conducted in the US, the Canadian market is distinct in several important ways. In Canada, federal regulations require premarket approval for nicotinecontaining e-cigarettes, and no products have received approval in Canada to date. Although e-cigarettes with nicotine are widely available through vape shops and online, supermarkets and other 
conventional retail outlets typically sell non-nicotine-containing e-cigarettes. At the time of the study, non-nicotine e-cigarettes could be legally purchased by minors, and accounted for a greater proportion of the e-cigarette market than in many other countries. ${ }^{4}$ In addition, there is little advertising or marketing for ecigarettes in traditional media outlets in Canada, in contrast to the situation in the US and the UK. ${ }^{17}$ Canada's distinct regulatory environment may have important implications for e-cigarette use among youth and its association with smoking behaviour. For example, the greater prevalence of non-nicotine-containing ecigarettes may be less likely to promote smoking behaviour, given the lack of nicotine exposure.

We sought to examine e-cigarette use in a large longitudinal sample of Canadian youth, including the extent to which e-cigarette use was associated with cigarette-smoking initiation at 1-year follow-up.

\section{Methods}

The COMPASS study is an ongoing cohort study that collects longitudinal data from a convenience sample of secondary school students (grades 9-12) in Ontario and Alberta, Canada. A full description of the design and methods of the study is available. ${ }^{18-20}$ For the current study, we assessed e-cigarette use at baseline (year 2 of the COMPASS study, in 2013/14), as well as at 1-year follow-up (year 3 of the study, in 2014/15).

Schools were recruited for the survey through purposefully selected school boards that met the following eligibility criteria: English-speaking, with at least 100 students in each of grades 9 through 12, and permitted the use of active-information, passiveconsent parental permission protocols (whereby information letters are mailed to the parents or guardians, who are asked to contact the researcher to decline their child's participation). Within each school, all students were invited to participate. A more detailed description of study enrolment is provided in Appendix 1 (available at www.cmaj .ca/lookup/suppl/doi:10.1503/cmaj.161002/-/DC1).

\section{Measures}

Data for all measures were collected using paper-based surveys administered by teachers, under the supervision of study research assistants. Sociodemographic measures included age, sex, race/ethnicity ("How would you describe yourself?" with the following "Mark all that apply" options: white, black, Asian, Aboriginal [First Nations, Metis, Inuit], Latin American/Hispanic and other; those selecting more than 1 option were categorized as "other/mixed") and spending money ("About how much money do you usually get each week to spend on yourself or to save? [Remember to include all money from allowances and jobs like babysitting, delivering papers, etc.]" with options of zero, $\$ 1$ to $\$ 5, \$ 6$ to $\$ 10, \$ 11$ to $\$ 20, \$ 21$ to $\$ 40, \$ 41$ to $\$ 100$, more than $\$ 100$ and "I do not know how much money I get each week").

We defined past 30-day cigarette use as 1 or more days in response to the question, "On how many of the last 30 days did you smoke one or more cigarettes?" We first classified smoking status into 6 categories: current daily smokers, who had smoked at least 100 cigarettes (lifetime) and had smoked every day of the past 30 days; current occasional smokers, who had smoked at least 100 cigarettes (lifetime) and had smoked on at least 1 day, but fewer than 30 days, of the past 30 days; former smokers, who had smoked at least 100 cigarettes (lifetime), but had not smoked in the last 30 days; experimental smokers, who had smoked a whole cigarette, but less than 100 cigarettes (lifetime); puffers, who had tried smoking but had not smoked a whole cigarette; and those who reported never having tried smoking. We then further classified the "never tried" group as susceptible or not susceptible to smoking, where susceptibility was defined as the absence of a firm commitment not to smoke (i.e., any response other than "definitely not") on all 3 of the following susceptibility questions: "Do you think in the future you might try smoking cigarettes?" "If one of your best friends was to offer you a cigarette, would you smoke it?" and "At any time during the next year do you think you will smoke a cigarette?"

We created a variable for smoking initiation using the measure of ever having smoked a whole cigarette ("Have you ever smoked a whole cigarette?") at baseline and follow-up: "never smokers" were those who responded "No" at both waves, and "initiators" were those who responded "No" at baseline but "Yes" at followup; those who had already smoked at baseline were excluded. We also created a variable for daily smoking initiation, using the measure of ever having smoked daily for 7 consecutive days ("Have you ever smoked every day for at least 7 days in a row?") at baseline and follow-up: "never daily smokers" were those who responded "No" at both waves, and "daily initiators" were those who responded "No" at baseline but "Yes" at follow-up; those who had already smoked every day for 7 days at baseline were excluded.

We assessed past 30-day use of e-cigarettes by asking, "In the last 30 days, did you use any of the following? (Mark all that apply)," with a list of products, including "e-cigarettes (electronic cigarettes that look like cigarettes/cigars, but produce vapour instead of smoke)."

\section{Statistical analysis}

All of the analyses were conducted using SAS version 9.4 (SAS Institute).

For cross-sectional analyses, we used $\chi^{2}$ tests to examine differences between survey years in past-month e-cigarette and cigarette use, as well as differences in characteristics between the single-wave and longitudinal samples. To examine variables associated with past 30-day use of e-cigarettes in the baseline sample, we estimated a generalized linear mixed-effects (GLM) model, including covariables for age, sex, race/ethnicity, spending money, smoking status (as fixed effects) and a random effect of school (to account for student clustering within schools). We also estimated separate GLM models for each covariable to generate unadjusted estimates.

For the longitudinal analyses, to examine cigarette-smoking initiation between baseline and follow-up, we estimated a GLM for the linked sample at follow-up (among those who had never smoked a whole cigarette at baseline), accounting for student clustering within schools by entering school as a random effect, and including the past-wave covariables (i.e., baseline values) of age, sex, race/ethnicity, spending money and past 30-day ecigarette use as fixed effects. We estimated a similar GLM model 
using daily cigarette-smoking initiation between baseline and follow-up (among those who had never smoked daily for 7 days at baseline). We excluded participants with missing data from the models on a case-wise basis.

\section{Ethics approval}

The COMPASS study was reviewed by and received ethics clearance from the University of Waterloo Office of Research Ethics and appropriate school board review panels.

\section{Results}

\section{Samples}

The COMPASS study was conducted in 89 secondary schools at baseline (79 in Ontario and 10 in Alberta) and 87 secondary schools at follow-up (78 in Ontario and 9 in Alberta); 86 schools (77 in Ontario and 9 in Alberta) participated at both baseline and follow-up. Figure 1 shows sample participation in each wave; 19310 students who provided data for both waves were included in the longitudinal analysis.

Table 1 shows the baseline characteristics of the overall and longitudinal samples. Students in the longitudinal sample, who provided data at both waves, were significantly more likely to be female, to report their race/ethnicity as white and to report lower levels of smoking and e-cigarette use, and were significantly different on age (younger at baseline) and spending money (less at baseline) compared with students who provided data at only 1 wave ( $p<0.001$ for all comparisons).

\section{Cross-sectional results: e-cigarette prevalence and predictors}

Among all students surveyed ( $n=44163$ at baseline; $n=41262$ at follow-up), prevalence of past 30-day e-cigarette use increased significantly from baseline to follow-up (7.2\% v. 9.7\%, $p<0.001)$. Past 30-day cigarette smoking decreased slightly between baseline and follow-up (11.4\% v. $10.8 \%, p=0.02)$.

Use of e-cigarettes was strongly associated with cigarette smoking: at baseline, $31.5 \%$ of students who had smoked a cigarette in the past 30 days also reported using an e-cigarette over the same period, compared with $5.0 \%$ of students who had not smoked in the past 30 days. After multivariable adjustment (and controlling for the effect of school), age, sex, race/ethnicity, spending money and smoking status were significantly associated with past 30-day e-cigarette use in the baseline sample (Table 2).

\section{Longitudinal results: initiation of cigarette smoking by follow-up}

Respondents who had already smoked a whole cigarette at baseline $(n=1992)$ were excluded from the analysis of initiation of cigarette smoking. Overall, $8.4 \%$ of the longitudinal sample who had "never smoked" at baseline had smoked a whole cigarette by 1-year follow-up. Students who reported past 30-day e-cigarette use at baseline were significantly more likely to initiate smoking at follow-up (adjusted odds ratio [OR] 2.12, 95\% confidence interval [Cl] 1.68-2.66) (Table 3). Smoking initiation was more likely among males (adjusted OR 1.44, 95\% Cl 1.28-1.62)

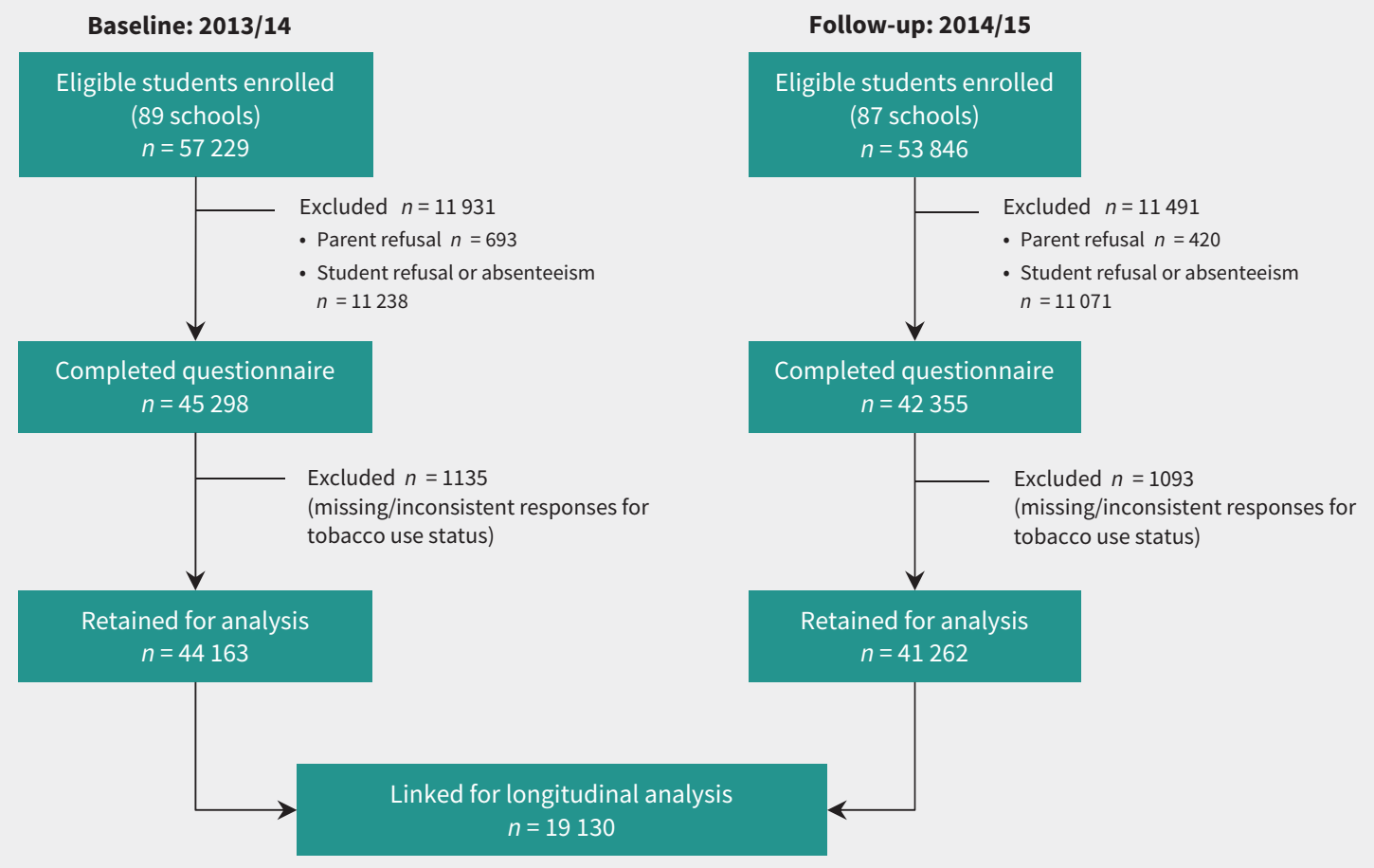

Figure 1: Respondent participation in the COMPASS study, 2013/14 and 2014/15 waves. 
and those with more spending money $(\$ 21-\$ 100$ v. $\$ 0$ per week, adjusted OR 1.25, 95\% Cl 1.04-1.50; > \$100 v. \$0 per week, adjusted OR 1.53, 95\% Cl 1.22-1.91). Compared with nonsusceptible never-smokers, smoking initiation was more likely among those who were susceptible to smoking (adjusted OR 3.86, 95\% $\mathrm{Cl} 3.36-4.43)$ and those who had already tried smoking less than a whole cigarette (adjusted OR 13.56, 95\% Cl 11.60-15.85).
The analysis of daily smoking initiation excluded respondents who had already smoked every day for 7 days at baseline $(n=619)$. Overall, $3.7 \%$ of the longitudinal sample who had never "smoked every day for 7 days in a row" at baseline had done so by 1-year follow-up. Correlates of daily smoking initiation at follow-up (Table 4) were similar to those for ever-smoking initiation, including e-cigarette use at baseline (adjusted OR 1.79, 95\% Cl 1.41-2.28).

\section{Table 1: Baseline characteristics of the overall COMPASS sample (2013/14) and}

respondents followed longitudinally to 2014/15, Ontario and Alberta, Canada

\begin{tabular}{|c|c|c|c|}
\hline \multirow[b]{2}{*}{ Characteristic (at baseline) } & \multicolumn{2}{|c|}{ Sample; no. (\%) of participants } & \multirow[b]{2}{*}{$p$ value $†$} \\
\hline & $\begin{array}{l}\text { Overall sample } \\
n=44163\end{array}$ & $\begin{array}{l}\text { Longitudinal sample* } \\
\qquad n=19310\end{array}$ & \\
\hline Age, yr & & & $<0.001$ \\
\hline$\leq 14$ & $9743(22.1)$ & $6255(32.4)$ & \\
\hline 15 & $11283(25.6)$ & $6517(33.7)$ & \\
\hline 16 & $11175(25.3)$ & $5238(27.1)$ & \\
\hline 17 & $9122(20.7)$ & $1198(6.2)$ & \\
\hline$\geq 18$ & $2840(6.4)$ & $102(0.5)$ & \\
\hline Sex & & & $<0.001$ \\
\hline Female & $21901(49.6)$ & $10303(53.4)$ & \\
\hline Male & $22262(50.4)$ & $9007(46.6)$ & \\
\hline Race/ethnicitył & & & $<0.001$ \\
\hline White & $32886(74.8)$ & $14940(77.7)$ & \\
\hline Black & $1689(3.8)$ & $603(3.1)$ & \\
\hline Asian & $2241(5.1)$ & $979(5.1)$ & \\
\hline Aboriginal & $1546(3.5)$ & $478(2.5)$ & \\
\hline Latin American/Hispanic & $830(1.9)$ & $305(1.6)$ & \\
\hline Other/mixed & $4759(10.8)$ & $1929(10.0)$ & \\
\hline Spending money, \$ & & & $<0.001$ \\
\hline 0 & $7046(16.0)$ & $3605(18.7)$ & \\
\hline $1-20$ & $12680(28.7)$ & $6594(34.1)$ & \\
\hline $21-100$ & $11749(26.6)$ & $4650(24.1)$ & \\
\hline$>100$ & $6994(15.8)$ & $1850(9.6)$ & \\
\hline Don't know/not stated & $5694(12.9)$ & $2611(13.5)$ & \\
\hline Smoking status & & & $<0.001$ \\
\hline Never tried, not susceptible & $22187(50.2)$ & $11075(57.4)$ & \\
\hline Never tried, susceptible & $9537(21.6)$ & $4719(24.4)$ & \\
\hline Puffer & $4465(10.1)$ & $1524(7.9)$ & \\
\hline Experimental smoker & $4819(10.9)$ & $1447(7.5)$ & \\
\hline Former smoker & $372(0.8)$ & $90(0.5)$ & \\
\hline Current occasional smoker & $1409(3.2)$ & $263(1.4)$ & \\
\hline Current daily smoker & $1374(3.1)$ & $192(1.0)$ & \\
\hline
\end{tabular}


Longitudinal results: change in smoking status at follow-up Transitions between all smoking status groups from baseline (2013/14) to follow-up (2014/15) are shown in Appendix 2 (available at www.cmaj.ca/lookup/suppl/doi:10.1503/cmaj.161002/-/ DC1). Among the 455 respondents who were current smokers at baseline (192 daily, 263 occasional), 39 (8.6\%) were former smokers at follow-up and 349 (76.7\%) were current smokers; the remaining $67(14.7 \%)$ were classified as having a nonsmoking status that was inconsistent with having been a current smoker (and were excluded from further analysis). Of the 39 respondents

Table 2: Past 30-day e-cigarette use and correlates in the COMPASS baseline sample (2013/14), Ontario and Alberta, Canada ( $n=44163)$

\begin{tabular}{|c|c|c|c|}
\hline \multirow[b]{2}{*}{ Characteristic } & \multirow{2}{*}{$\begin{array}{l}\text { No. (\%) within category } \\
\text { with past } 30 \text {-day } \\
\text { e-cigarette use at baseline }\end{array}$} & \multicolumn{2}{|c|}{ OR $(95 \% \mathrm{CI})$} \\
\hline & & Unadjusted * & Adjusted $†$ \\
\hline \multicolumn{4}{|l|}{ Age, yr } \\
\hline$\leq 14$ & $458 / 9743(4.7)$ & 1.0 (ref) & 1.0 (ref) \\
\hline 15 & $765 / 11283(6.8)$ & $1.46(1.29-1.64)$ & $1.07(0.94-1.22)$ \\
\hline 16 & $962 / 11175$ (8.6) & $1.88(1.67-2.11)$ & $1.01(0.89-1.15)$ \\
\hline 17 & $738 / 9122(8.1)$ & $1.75(1.55-1.98)$ & $0.73(0.63-0.84)$ \\
\hline$\geq 18$ & $243 / 2840(8.6)$ & $1.87(1.59-2.21)$ & $0.62(0.51-0.74)$ \\
\hline \multicolumn{4}{|l|}{ Sex } \\
\hline Female & $1117 / 21901(5.1)$ & 1.0 (ref) & 1.0 (ref) \\
\hline Male & $2049 / 22262(9.2)$ & $1.91(1.77-2.06)$ & $1.70(1.57-1.85)$ \\
\hline \multicolumn{4}{|l|}{ Race/ethnicity } \\
\hline White & $2236 / 32886(6.8)$ & 1.0 (ref) & 1.0 (ref) \\
\hline Black & $152 / 1689(9.0)$ & $1.48(1.24-1.76)$ & $1.10(0.91-1.34)$ \\
\hline Asian & $96 / 2241(4.3)$ & $0.60(0.48-0.74)$ & $0.68(0.54-0.86)$ \\
\hline Aboriginal & $139 / 1546(9.0)$ & $1.34(1.11-1.63)$ & $0.68(0.55-0.84)$ \\
\hline Latin American/Hispanic & $61 / 830(7.3)$ & $1.14(0.87-1.48)$ & $0.89(0.66-1.18)$ \\
\hline Other/mixed & $467 / 4759(9.8)$ & $1.52(1.37-1.69)$ & $1.21(1.08-1.36)$ \\
\hline \multicolumn{4}{|l|}{ Spending money, \$ } \\
\hline 0 & $312 / 7046(4.4)$ & 1.0 (ref) & 1.0 (ref) \\
\hline $1-20$ & $748 / 12680(5.9)$ & $1.37(1.19-1.57)$ & $1.33(1.15-1.54)$ \\
\hline $21-100$ & $968 / 11749(8.2)$ & $1.94(1.70-2.22)$ & $1.63(1.42-1.89)$ \\
\hline$>100$ & $813 / 6994$ (11.6) & $2.86(2.49-3.28)$ & $1.80(1.54-2.09)$ \\
\hline Don't know/not stated & $325 / 5694(5.7)$ & $1.29(1.10-1.51)$ & $1.21(1.02-1.44)$ \\
\hline \multicolumn{4}{|l|}{ Smoking status } \\
\hline Never tried, not susceptible & $343 / 22187(1.5)$ & 1.0 (ref) & 1.0 (ref) \\
\hline Never tried, susceptible & $412 / 9537(4.3)$ & $2.83(2.45-3.28)$ & $2.76(2.38-3.20)$ \\
\hline Puffer & $468 / 4465(10.5)$ & $7.85(6.79-9.07)$ & $7.79(6.73-9.01)$ \\
\hline Experimental smoker & $924 / 4819$ (19.2) & $16.03(14.07-18.26)$ & $15.64(13.69-17.87)$ \\
\hline Former smoker & $49 / 372(13.2)$ & $10.84(7.85-14.96)$ & $10.48(7.55-14.54)$ \\
\hline Current occasional smoker & $409 / 1409(29.0)$ & $29.52(25.15-34.66)$ & $28.79(24.41-33.96)$ \\
\hline Current daily smoker & $561 / 1374(40.8)$ & $50.03(42.82-58.45)$ & $48.85(41.56-57.43)$ \\
\hline \multicolumn{4}{|c|}{$\begin{array}{l}\text { Note: } \mathrm{Cl}=\text { confidence interval, } \mathrm{OR}=\text { odds ratio. } \\
\text { *From separate generalized linear mixed models for using an e-cigarette in the past } 30 \text { days, including only the listed covariable, with } \\
\text { school as a random effect }(n=44163 \text { for all models except race/ethnicity; } n=43951 \text { for race/ethnicity model). } \\
\text { tFrom a generalized linear mixed model for using an e-cigarette in the past } 30 \text { days, including all covariables in the table and school } \\
(n=89) \text { as a random effect }(n=43951) \text {. } \\
\text { fInformation on race/ethnicity was missing for } 212 \text { participants; these were excluded from the model. }\end{array}$} \\
\hline
\end{tabular}


who became former smokers, 10 (25.6\%) reported past 30-day e-cigarette use at baseline and $6(15.4 \%)$ reported past 30-day e-cigarette use at follow-up. Of the 349 respondents who remained current smokers, $116(33.2 \%)$ reported past 30-day e-cigarette use at baseline and $130(37.2 \%)$ reported past 30-day e-cigarette use at follow-up.

\section{Interpretation}

The current study is among the largest longitudinal studies of ecigarette use and cigarette-smoking initiation to date. The findings are consistent with those of other surveys conducted in Canada, which have shown increasing rates of e-cigarette use among youth:

\section{Table 3: Between-wave smoking initiation* in the CoMPASS follow-up (2014/15) longitudinal}

sample, Ontario and Alberta, Canada $(n=17318)$

\begin{tabular}{|c|c|c|c|}
\hline \multirow[b]{2}{*}{ Characteristic (at baseline) } & \multirow{2}{*}{$\begin{array}{c}\text { No. (\%) within category } \\
\text { who initiated smoking } \\
\text { by follow-up }\end{array}$} & \multicolumn{2}{|c|}{ OR $(95 \% \mathrm{CI})$} \\
\hline & & Unadjusted $\dagger$ & Adjusted¥ \\
\hline \multicolumn{4}{|l|}{ Age, yr } \\
\hline$\leq 14$ & $436 / 5936(7.3)$ & 1.0 (ref) & 1.0 (ref) \\
\hline 15 & $511 / 5845(8.7)$ & $1.23(1.07-1.41)$ & $1.08(0.94-1.25)$ \\
\hline 16 & $397 / 4464(8.9)$ & $1.27(1.10-1.47)$ & $1.04(0.89-1.21)$ \\
\hline$\geq 17 \S$ & $105 / 1073(9.8)$ & $1.43(1.13-1.79)$ & $1.02(0.80-1.32)$ \\
\hline \multicolumn{4}{|l|}{ Sex } \\
\hline Female & $683 / 9289(7.4)$ & 1.0 (ref) & 1.0 (ref) \\
\hline Male & $766 / 8029(9.5)$ & $1.37(1.22-1.52)$ & $1.44(1.28-1.62)$ \\
\hline \multicolumn{4}{|l|}{ Race/ethnicityฯ } \\
\hline White & $1143 / 13423(8.5)$ & 1.0 (ref) & 1.0 (ref) \\
\hline Black & $42 / 562(7.5)$ & $1.00(0.72-1.40)$ & $1.03(0.72-1.45)$ \\
\hline Asian & $44 / 925(4.8)$ & $0.62(0.45-0.85)$ & $0.67(0.72-0.93)$ \\
\hline Aboriginal & $58 / 328(17.7)$ & $2.04(1.50-2.77)$ & $1.29(0.93-1.80)$ \\
\hline Latin American/Hispanic & $21 / 274(7.7)$ & $0.98(0.62-1.54)$ & $0.71(0.44-1.15)$ \\
\hline Other/mixed & $140 / 1735(8.1)$ & $1.00(0.83-1.20)$ & $0.95(0.78-1.16)$ \\
\hline \multicolumn{4}{|l|}{ Spending money, \$ } \\
\hline 0 & $224 / 3342(6.7)$ & 1.0 (ref) & 1.0 (ref) \\
\hline $1-20$ & $454 / 6048(7.5)$ & $1.14(0.96-1.34)$ & $1.00(0.84-1.20)$ \\
\hline $21-100$ & $416 / 4077(10.2)$ & $1.57(1.32-1.86)$ & $1.25(1.04-1.50)$ \\
\hline$>100$ & $193 / 1473(13.1)$ & $2.05(1.67-2.52)$ & $1.53(1.22-1.91)$ \\
\hline Don't know/not stated & $162 / 2378(6.8)$ & $1.01(0.82-1.25)$ & $1.02(0.82-1.28)$ \\
\hline \multicolumn{4}{|l|}{ Smoking status } \\
\hline Never tried, not susceptible & $364 / 11075(3.3)$ & 1.0 (ref) & 1.0 (ref) \\
\hline Never tried, susceptible & $562 / 4719(11.9)$ & $3.95(3.44-4.53)$ & $3.86(3.36-4.43)$ \\
\hline Puffer & $523 / 1624(34.3)$ & $15.08(12.97-17.53)$ & $13.56(11.60-15.85)$ \\
\hline \multicolumn{4}{|l|}{ Past 30-day e-cigarette use } \\
\hline No & $1313 / 16831(7.8)$ & 1.0 (ref) & 1.0 (ref) \\
\hline Yes & $136 / 487(27.9)$ & $4.81(3.90-5.94)$ & $2.12(1.68-2.66)$ \\
\hline \multicolumn{4}{|c|}{$\begin{array}{l}\text { Note: } \mathrm{Cl}=\text { confidence interval, } \mathrm{OR}=\text { odds ratio. } \\
\text { *Among those who had never smoked a whole cigarette at baseline, but had smoked a whole cigarette at follow-up; } n=1992 \text { had already } \\
\text { initiated smoking at baseline and were excluded from this measure. } \\
\text { tFrom separate generalized linear mixed models for initiating smoking between waves, including only the listed covariable, with school as a } \\
\text { random effect ( } n=17318 \text { for all models except race/ethnicity; } n=17247 \text { for race/ethnicity model). } \\
\text { tFrom a generalized linear mixed model for initiating smoking between waves, including the covariables in the table, with school }(n=86) \text { as } \\
\text { a random effect ( }(n=17247) \text {. } \\
\text { \$Categories for age } 17 \text { and age } \geq 18 \text { were combined because of low numbers for the latter category }(n=8) \text {. } \\
\text { ฯIInformation on race/ethnicity was missing for } 71 \text { participants; these were excluded from the multivariable model. }\end{array}$} \\
\hline
\end{tabular}


in our study, about $10 \%$ of youth reported using an e-cigarette in the past 30 days, compared with national estimates of $3 \%$ in 2013.,7

In the cross-sectional analysis, e-cigarette use was associated with cigarette-smoking behaviour in a dose-response fashion. Differences in past 30-day e-cigarette use were observed even among lower thresholds of smoking behaviour, including between "puffers" and never smokers, and by smoking susceptibility. We did not assess reasons for e-cigarette use among youth smokers; however, research with adults has indicated that smokers use e-cigarettes for a variety of reasons other than quitting,

Table 4: Between-wave daily smoking initiation* in the COMPASS follow-up (2014/15) longitudinal sample, Ontario and Alberta, Canada $(n=18691)$

\begin{tabular}{|c|c|c|c|}
\hline \multirow[b]{2}{*}{ Characteristic (at baseline) } & \multirow{2}{*}{$\begin{array}{l}\text { No. }(\%) \text { within category } \\
\text { who initiated daily } \\
\text { smoking by follow-up }\end{array}$} & \multicolumn{2}{|c|}{ OR $(95 \% \mathrm{Cl})$} \\
\hline & & Unadjusted $\dagger$ & Adjusted $\ddagger$ \\
\hline \multicolumn{4}{|l|}{ Age, yr } \\
\hline$\leq 14$ & $204 / 6179(3.3)$ & 1.0 (ref) & 1.0 (ref) \\
\hline 15 & $219 / 6298(3.5)$ & $1.04(0.86-1.27)$ & $0.74(0.60-0.92)$ \\
\hline 16 & $206 / 4997(4.1)$ & $1.27(1.04-1.55)$ & $0.70(0.55-0.88)$ \\
\hline$\geq 17 \S$ & $58 / 1217(4.8)$ & $1.46(1.07-1.98)$ & $0.73(0.51-1.03)$ \\
\hline \multicolumn{4}{|l|}{ Sex } \\
\hline Female & $320 / 9996(3.2)$ & 1.0 (ref) & 1.0 (ref) \\
\hline Male & $367 / 8695(4.2)$ & $1.36(1.17-1.59)$ & $1.36(1.15-1.62)$ \\
\hline \multicolumn{4}{|l|}{ Race/ethnicityฯ } \\
\hline White & $525 / 14488$ (3.6) & 1.0 (ref) & 1.0 (ref) \\
\hline Black & $21 / 585(3.6)$ & $1.23(0.78-1.94)$ & $1.30(0.78-2.15)$ \\
\hline Asian & $11 / 965(1.1)$ & $0.38(0.21-0.70)$ & $0.45(0.24-0.85)$ \\
\hline Aboriginal & $43 / 406(10.6)$ & $2.49(1.75-3.54)$ & $1.42(0.96-2.09)$ \\
\hline Latin American/Hispanic & $11 / 296(3.7)$ & $1.21(0.65-2.23)$ & $1.03(0.54-1.98)$ \\
\hline Other/mixed & $75 / 1876(4.0)$ & $1.21(0.94-1.56)$ & $1.09(0.82-1.43)$ \\
\hline \multicolumn{4}{|l|}{ Spending money, \$ } \\
\hline 0 & $95 / 3513(2.7)$ & 1.0 (ref) & 1.0 (ref) \\
\hline $1-20$ & $219 / 6436(3.4)$ & $1.26(0.99-1.62)$ & $1.07(0.81-1.39)$ \\
\hline $21-100$ & $182 / 4480(4.1)$ & $1.49(1.15-1.92)$ & $1.03(0.78-1.36)$ \\
\hline$>100$ & $109 / 1731(6.3)$ & $2.24(1.69-2.99)$ & $1.16(0.84-1.60)$ \\
\hline Don't know/not stated & $82 / 2531(3.2)$ & $1.20(0.89-1.62)$ & $1.15(0.83-1.60)$ \\
\hline \multicolumn{4}{|l|}{ Smoking status } \\
\hline Never tried, not susceptible & $83 / 11075(0.7)$ & 1.0 (ref) & 1.0 (ref) \\
\hline Never tried, susceptible & $129 / 4719(2.7)$ & $3.69(2.79-4.87)$ & $3.58(2.70-4.73)$ \\
\hline Puffer & $106 / 1514(7.0)$ & $9.81(7.31-13.16)$ & $9.33(6.91-12.59)$ \\
\hline Experimental smoker & $308 / 1274(24.2)$ & $40.80(31.65-52.60)$ & $37.73(28.91-49.25)$ \\
\hline Former smoker & $13 / 29(44.8)$ & 96.81 (44.52-210.49) & 94.99 (43.19-208.92) \\
\hline Current occasional smoker & $48 / 80(60.0)$ & $193.01(116.72-319.16)$ & $157.54(93.59-265.19)$ \\
\hline \multicolumn{4}{|l|}{ Past 30-day e-cigarette use } \\
\hline No & $551 / 17911(3.1)$ & 1.0 (ref) & 1.0 (ref) \\
\hline Yes & $136 / 780(17.4)$ & $6.97(5.65-8.60)$ & $1.79(1.41-2.28)$ \\
\hline \multicolumn{4}{|c|}{$\begin{array}{l}\text { Note: } \mathrm{Cl}=\text { confidence interval, } \mathrm{OR}=\text { odds ratio. } \\
\text { *Among those who had never smoked every day for } 7 \text { days at baseline, but had smoked every day for } 7 \text { days at follow-up; } n=619 \text { had already } \\
\text { initiated daily smoking at baseline and were excluded from this measure. } \\
\text { †From separate generalized linear mixed models for initiating daily smoking between waves, including only the listed covariable, with school as } \\
\text { a random effect ( } n=18691 \text { for all models except race/ethnicity; } n=18616 \text { for race/ethnicity model). } \\
\text { †From a generalized linear mixed model for initiating daily smoking between waves, including the covariables in the table, with school }(n=86) \\
\text { as a random effect ( } n=18616) \text {. } \\
\text { \$Categories for age } 17 \text { and age } \geq 18 \text { were combined because of low numbers for the latter category }(n=5) \text {. } \\
\text { IInformation on race/ethnicity was missing for } 75 \text { participants; these were excluded from the multivariable model. }\end{array}$} \\
\hline
\end{tabular}


including for use in places where smoking is not allowed and to cut back on, but not quit, smoking. ${ }^{21}$ There is a need for similar data on reasons for use from population-based studies of youth.

Youth who reported e-cigarette use in the past 30 days at baseline were more likely to initiate cigarette smoking and more likely to report having smoked daily at follow-up, even after adjustment for a range of other factors at baseline. These findings are consistent with prior longitudinal studies. ${ }^{13,14,16}$ It is unclear the extent to which this association is causal. At the individual level, e-cigarettes may be causally related to cigarette smoking because they provide early exposure to nicotine or greater exposure to environmental risk factors, including greater exposure to smokers or certain social settings. E-cigarette use may also help to "re-normalize" smoking by promoting more positive normative beliefs about nicotine use and smoking, which are important predictors of uptake. ${ }^{22}$ Alternatively, both e-cigarettes and cigarette smoking could be the result of unmeasured "common factors." studies to date have adjusted for factors such as sensationseeking, parental support and rebelliousness, and have found that the association with e-cigarette use persists; however, it is doubtful that any analysis can adequately control for the range of "common factors" that may account for the use of multiple nicotine products. ${ }^{14,15}$ Put more simply, youth who try e-cigarettes may be different from those who do not. ${ }^{24}$ The temporal order, whereby e-cigarette use precedes cigarette-smoking initiation, may be explained by the fact that e-cigarettes are more accessible than cigarettes to Canadian youth, and are therefore likely to be encountered and used first.

Despite a substantial increase in the prevalence of past 30-day e-cigarette use between baseline and follow-up, the prevalence of cigarette smoking decreased slightly in the overall sample. This pattern of data has previously been cited by proponents as evidence that e-cigarette use does not increase cigarettesmoking initiation and, subsequently, smoking rates; however, it is also possible that smoking rates might have decreased further in the absence of e-cigarette use. Regardless, the current pattern of data suggests that - if e-cigarettes do in fact increase smoking initiation - the impact to date has been insufficient to completely stall or reverse the decline in youth smoking in Canada.

\section{Limitations and strengths}

The current study is among the largest to examine e-cigarette use among youth. The study was conducted in only 2 Canadian provinces, and schools were not recruited using probabilitybased sampling methods; therefore, comparisons with national estimates should be made with caution. The study had several limitations common to longitudinal research, including nonrandom attrition at follow-up. All measures relied on self-report; however, the confidentiality of responses was emphasized, and self-reported measures of tobacco use have been previously validated using biochemical measures. ${ }^{25}$ The regulatory context of ecigarettes in Canada should also be noted when interpreting these findings. In particular, non-nicotine-containing ecigarettes account for a greater proportion of the e-cigarette market in Canada than in many other countries. ${ }^{3,26}$ Future research should examine the likelihood of smoking initiation with nicotine-containing versus non-nicotine-containing e-cigarettes. Finally, the current study examined only 1 dimension of ecigarettes and cigarette smoking (i.e., initiation): it did not have adequate statistical power to assess the impact of e-cigarette use on smoking cessation among youth smokers, which is a critically important determinant of the net public health impact of ecigarettes. However, we have reported raw data on e-cigarette use and subsequent smoking cessation.

\section{Conclusion}

The current study provides strong evidence that e-cigarettes are associated with initiation of cigarette smoking among youth; however, the controversy as to whether e-cigarette use "causes" cigarette-smoking initiation will undoubtedly persist. In fact, the findings from our study provide support for both sides of the debate. It is highly plausible that "common factors" account for a substantial proportion of increased cigarette-smoking initiation among e-cigarette users. At the same time, it would be foolhardy to dismiss the likelihood that early exposure to nicotine via ecigarettes increases smoking uptake. Attributing the relative importance of these 2 factors will not be straightforward, and represents a critical challenge to the research community. In the meantime, regulatory frameworks that succeed in shifting ecigarette use away from youth and concentrating their use among cigarette smokers for the purposes of smoking cessation are likely to have the greatest public health impact.

\section{References}

1. Reid JL, Hammond D, Rynard VL, et al. Tobacco use in Canada: patterns and trends, 2017 edition. Waterloo (ON): University of Waterloo, Propel Centre for Population Health Impact; 2017. Available: https://uwaterloo.ca/tobacco-use -canada/tobacco-use-canada-patterns-and-trends (accessed 2017 June 21).

2. Farsalinos KE, Poulas K, Voudris V, et al. Electronic cigarette use in the European Union: analysis of a representative sample of 27460 Europeans from 28 countries. Addiction 2016;111:2032-40.

3. Reid JL, Rynard VL, Czoli CD, et al. Who is using e-cigarettes in Canada? Nationally representative data on the prevalence of e-cigarette use among Canadians. Prev Med 2015;81:180-3.

4. Czoli CD, Reid JL, Rynard VL, et al. E-cigarettes in Canada - tobacco use in Can ada: patterns and trends, 2015 edition, special supplement. Waterloo (ON): University of Waterloo, Propel Centre for Population Health Impact; 2015. Available: https://uwaterloo.ca/tobacco-use-canada/e-cigarette-supplement (accessed 2017 June 21).

5. Carroll Chapman SL, Wu LT. E-cigarette prevalence and correlates of use among adolescents versus adults: a review and comparison. J Psychiatr Res 2014; 54:43-54.

6. Eastwood B, Dockrell MJ, Arnott D, et al. Electronic cigarette use in young people in Great Britain 2013-2014. Public Health 2015;129:1150-6.

7. Singh T, Arrazola RA, Corey CG, et al. Tobacco use among middle and high school students - United States, 2011-2015. MMWR Morb Mortal Wkly Rep 2016; 65:361-7.

8. Khoury M, Manlhiot C, Fan CPS, et al. Reported electronic cigarette use among adolescents in the Niagara region of Ontario. CMAJ 2016;188:794-800.

9. Barnett TE, Soule EK, Forrest JR, et al. Adolescent electronic cigarette use: associations with conventional cigarette and hookah smoking. Am J Prev Med 2015; 49:199-206.

10. Dutra LM, Glantz SA. Electronic cigarettes and conventional cigarette use among U.S. adolescents: a cross-sectional study [published erratum in JAMA Pediatr 2014;168:684]. JAMA Pediatr 2014;168:610-7. 
11. Arrazola RA, Singh T, Corey CG, et al.; Centers for Disease Control and Prevention (CDC). Tobacco use among middle and high school students - United States, 2011-2014. MMWR Morb Mortal Wkly Rep 2015;64:381-5.

12. Barrington-Trimis JL, Urman R, Leventhal AM, et al. E-cigarettes, cigarettes, and the prevalence of adolescent tobacco use. Pediatrics 2016;138:e20153983.

13. Leventhal AM, Strong DR, Kirkpatrick MG, et al. Association of electronic cigarette use with initiation of combustible tobacco product smoking in early adolescence. JAMA 2015;314:700-7.

14. Wills TA, Knight R, Sargent JD, et al. Longitudinal study of e-cigarette use and onset of cigarette smoking among high school students in Hawaii. Tob Control 2017;26:34-9.

15. Primack BA, Soneji S, Stoolmiller M, et al. Progression to traditional cigarette smoking after electronic cigarette use among US adolescents and young adults. JAMA Pediatr 2015;169:1018-23.

16. Barrington-Trimis JL, Urman R, Berhane $\mathrm{K}$, et al. E-cigarettes and future cigarette use. Pediatrics 2016;138:e20160379.

17. Hammond D, White CM, Czoli CD, et al. Retail availability and marketing of electronic cigarettes in Canada. Can J Public Health 2015;106:e408-12.

18. Bredin C, Leatherdale ST. Methods for linking COMPASS student-level data over time. COMPASS Tech Rep Ser Vol. 1, Issue 2. Waterloo (ON): University of Waterloo; 2013. Available: https://uwaterloo.ca/compass-system/publications /methods-linking-compass-student-level-data-over-time (accessed 2017 June 21).

19. Qian W, Battista K, Bredin C, et al. Assessing longitudinal data linkage results in the COMPASS study. COMPASS Tech Rep Ser Vol. 3, Issue 4. Waterloo (ON):
University of Waterloo; 2015. Available: https://uwaterloo.ca/compass-system/ publications/assessing-longitudinal-data-linkage-results-compass-study (accessed 2017 June 21).

20. Leatherdale ST, Brown KS, Caron V, et al. The COMPASS study: a longitudinal hierarchical research platform for evaluating natural experiments related to changes in school-level programs, policies and built environment resources. BMC Public Health 2014;14:331.

21. Shiplo S, Czoli CD, Hammond D. E-cigarette use in Canada: prevalence and patterns of use in a regulated market. BMJ Open 2015;5:e007971.

22. Preventing tobacco use among youth and young adults: a report of the Surgeon General. Atlanta: US Department of Health and Human Services, Centers for Disease Control and Prevention, National Center for Chronic Disease Prevention and Health Promotion, Office on Smoking and Health; 2012.

23. Morral AR, McCaffrey DF, Paddock SM. Reassessing the marijuana gateway effect. Addiction 2002;97:1493-504.

24. Niaura RS, Glynn TJ, Abrams DB. Youth experimentation with e-cigarettes: another interpretation of the data. JAMA 2014;312:641-2.

25. Wong SL, Shields M, Leatherdale S, et al. Assessment of validity of selfreported smoking status. Health Rep 2012;23:47-53.

26. Notice - to all persons interested in importing, advertising or selling electronic smoking products in Canada. Ottawa: Health Canada; 2009. Available: https:// www.canada.ca/en/health-canada/services/drugs-health-products/drug-products /applications-submissions/policies/persons-interested-importing-advertising -selling-electronic-smoking-products-canada.html (accessed 2017 June 21).

\section{Competing interests: None declared.}

This article has been peer reviewed.

Affiliation: School of Public Health and Health Systems, University of Waterloo, Waterloo, Ont.

Contributors: Scott Leatherdale was primarily responsible for the study design, with contributions from David Hammond. David Hammond and Jessica Reid were primarily responsible for the analysis and manuscript preparation. All of the authors interpreted the data and contributed to writing and revising the manuscript, had full access to all of the data (including statistical reports and tables) in the study, gave final approval of the version to be published and agree to be accountable for all aspects of the work.
Funding: The COMPASS study was supported by a bridge grant from the Canadian Institutes of Health Research (CIHR) Institute of Nutrition, Metabolism and Diabetes through the Obesity - Interventions to Prevent or Treat priority funding awards (OOP110788, awarded to Scott Leatherdale) and an operating grant from the CIHR Institute of Population and Public Health (MOP-114875, awarded to Scott Leatherdale). Additional support for this paper was provided by an Ontario Ministry of Health and Long-Term Care Health Systems Research Fund grant (06697, awarded to David Hammond), a CIHR New Investigator Award (awarded to David Hammond), a CIHR Doctoral Research Award - Frederick Banting and Charles Best Canada Graduate Scholarship (awarded to Adam
Cole) and CIHR Public Health Agency of Canada Chairs in Applied Public Health (awarded to David Hammond and Scott Leatherdale). The researchers are independent from all sources of funding; the study sponsors had no role in study design; the collection, analysis or interpretation of data; the writing of the article; or the decision to submit it for publication.

Acknowledgement: The authors thank Wei Qian for consulting on statistical methods.

Accepted: Aug. 4, 2017

Correspondence to: David Hammond, dhammond@uwaterloo.ca 